\title{
A Hybrid Non Blind Video Watermarking Scheme Based on DWPT and PCA
}

\author{
Shital Khaparde ${ }^{1}$, Shanta Sondor ${ }^{2}$ \\ ${ }^{1,2}$ Mumbai University, Department of Information and Technology, VES College, Mumbai, India
}

\begin{abstract}
This paper present a novel hybrid watermarking technique which combine two watermarking methods Discrete Wavelet Packet Transform (DWPT) and Principle Component Analysis (PCA). It embed a binary logo watermark into video frames. Combining the two transforms improved the performance of the watermarking algorithm. The performance can be evaluated in terms of the imperceptibility and robustness against various attacks such as contrast adjustment, noise addition and geometric attacks. The PeakSignal-To-Noise Ratio (PSNR) used to measure the visual quality of watermarked video. NC is the normalized correlation between the original and the extracted watermark. Experimental results has shown no visible difference between the watermarked frames and the original frames, and shown the robustness against a wide range of attacks such as Gaussian noise addition, histogram equalization, salt and paper noise, contrast adjustment, JPEG compression, cropping, resizing, rotation, frame dropping, frame averaging, frame swapping etc.
\end{abstract}

Keywords: Principal Component Analysis (PCA), Discrete Wavelet Packet Transform (DWPT), Peak-Signal-To-Noise Ratio (PSNR), Normalized correlation (NC).

\section{Introduction}

Digital content (audio, video, graphics, and images) can be easily copied, transmitted and distributed over networks. The expansion of the internet, making it the most powerful tool for information exchange today, as well as the numerous file sharing tools have made the distribution of copyrighted digital media files simple nowadays. DRM (Digital rights management) refers to the technologies or systems that protect and enforce the rights associated with the use of digital content. Digital Rights Management (DRM) systems aim to control access and actions that can be performed by users (both human and machine)[2]. DRM technologies have become very important in an increasingly networked world because it promises the owner of the file persistent control over the file even when the file leaves the owner's machine. There are various techniques used for copyrights protection by the DRM such as encryption, traitor tracing, fingerprinting and watermarking $[3,4]$.

In the literature, different digital video watermarking algorithms have been proposed. The [1] authors have implemented digital video watermarking based on DWT and PCA. The video frames are first decomposed using DWT and the binary watermark is embedded in the principal components of the low frequency wavelet coefficients. In "Video Watermarking based on DWT and PCA" [7] authors have implemented PCA to each block of the two bands (LL $\mathrm{HH}$ ) which result from DWT of every video frame. The watermark is embedded into the principal components of the LL blocks and $\mathrm{HH}$ blocks in different ways. This hybrid scheme given improved imperceptible and a robustness against some attacks. In "A robust discrete wavelet packet transform (DWPT) based on digital image watermarking using HVS characteristics" [10] authors have selected the DWPT decomposition to utilize all high frequency components in order to make the watermarking more imperceptible using Coif5 as wavelet basis. According to the characteristics of Human Visual System (HVS), human eyes are less sensitive in high frequency bands. In "Block Based Video Watermarking Scheme Using 2D level DWT and PCA" [5] authors have decomposed each video frame into sub-images using 2 level DWT then the PCA transformation is applied for each block in the two bands LL and HH. The watermark is embedded into the maximum coefficient of the PCA block of the two bands. The 2 level DWT gives more details information of each bands which helps in more good quality of video frame after embedding watermark. There are other schemes also based on these transforms $[6,8,9]$

In this paper, we proposed video watermarking scheme based on combining two transformations DWPT and PCA. The proposed scheme combines both the DWPT and PCA to develop a new hybrid non-blind scheme that is resistant to a variety of attacks $[1,10]$. The binary watermark image used to embed into the video frames. Each video frame is decomposed into all frequency sub-bands using 2 level DWPT and then the PCA transformation is applied for each block in the low frequency bands. The watermark is embedded into the maximum coefficient of the PCA block of the band.

The rest of the paper is organized as follows: section 2 presents the proposed watermarking scheme. Section 3 introduces the experimental results and finally section 4 concludes the paper.

\section{The Proposed Scheme}

The proposed watermarking scheme is based on combining two transformations; the DWPT and the PCA. The watermark is embedded in the luminance component of each frame of the un coded video. Each video frame is decomposed into four sub-bands using 2 level DWPT then the PCA transformation is applied for each block in the low frequency bands. The scheme consists of four main modules and there are explained in detail as follows: 


\section{International Journal of Science and Research (IJSR) \\ ISSN (Online): 2319-7064}

Index Copernicus Value (2013): 6.14 | Impact Factor (2015): 6.391

\subsection{Module 1: Embedding watermark}

In this algorithm we first divide the video divided into frames and it is converted from RGB to YUV components. Then the 2 level DWPT is apply on the frames to break it into $\mathrm{cA}, \mathrm{cH}$, $\mathrm{cV} \& \mathrm{cD}$ sub bands as shown in fig 1 . The $\mathrm{cA}$ sub band is divided into sub blocks and on each sub block the PCA is applied to get principal component and on this PCA component the binary watermark is embedded. After embedding the watermark inverse of PCA and inverse of DWPT is carried out and the video frame is converted back to RGB from YUV. In this way embedding process is completed.

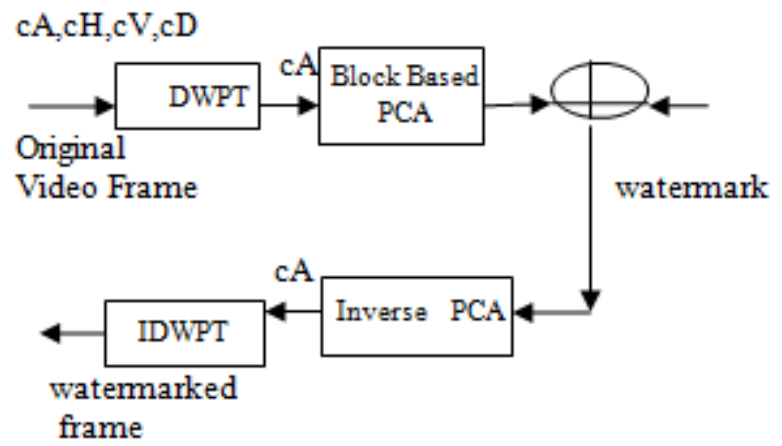

Figure 1: Embedding procedure

\subsection{Module 2: Extracting watermark}

In this algorithm the embedded watermark video is taken as an input and watermark extracted from this video. The procedure of this is same as the embedding process here also the video is converted from RGB to YUV component and then the 2 level DWPT is apply on it to break the video into $\mathrm{cA}, \mathrm{cH}, \mathrm{cV} \& \mathrm{cD}$ sub bands as shown in fig 2. The cA sub band is the divided into sub blocks and on each sub block the PCA is applied to get principal component of it. After this the binary watermark's bits are extracted from the principle component. We compared the extracted watermark with the original watermark by calculating NC value.

\subsection{Module 3: Verifying watermark against various attacks}

In this we have extracted the watermark from the embedded watermark video and the various attacks like JPEG coding, Gaussian noise addition, histogram equalization, salt and pepper noise, contrast adjustment, frame dropping, frame swapping, frame averaging, cropping, resizing, and rotation, etc. are performed on it.

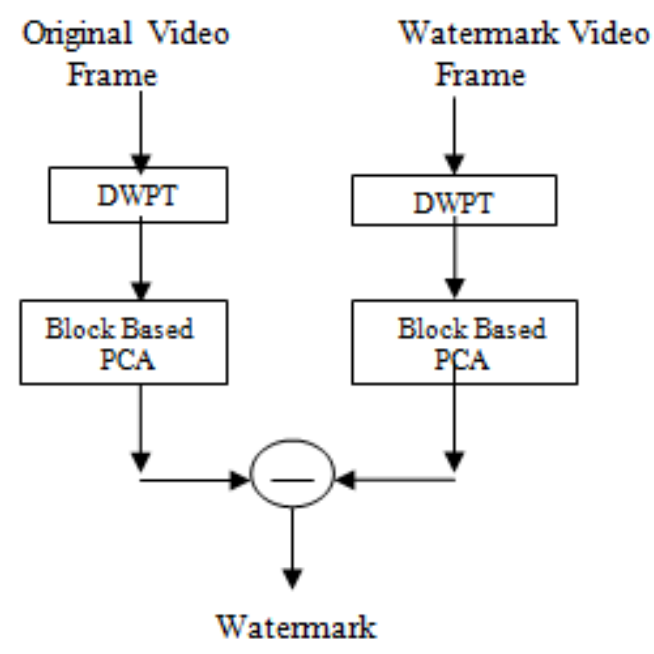

Figure 2: Extraction procedure

After performing various attacks the effect of attacks on the watermark is seen by calculating the NC values.

\subsection{Module 4: Calculating PSNR and NC values}

The performance of the scheme has been evaluated in terms of the imperceptibility and robustness against various attacks. The PSNR is used to measure the visual quality of watermarked and robustness i.e the existence of the watermark after the attack is measured by NC.

\section{Experimental Results}

We have taken three videos to test the proposed scheme, they are namely Pepsi, Lake [1] and Foreman [7]. The video is divided into 50 frame of size 256x256. Only the luminance components of the all 50 frames are watermarked during the test procedures. The watermark is a binary logo image with size $32 \times 32$ is taken. The grayscale watermark is converted to binary before embedding into the video frame. After embedding, the watermark is extracted and its NC value is calculated with respect to the original watermark. To test the robustness of the watermark various attacks are performed on the watermark embedded video frame. The average PSNR value of 50 watermarked frames of Pepsi Video is $40.05 \mathrm{~dB}$ as shown in fig.3. In this case, the watermarked frame appears visually identical to the original.

Table 1: PSNR values for referred videos

\begin{tabular}{|c|c|c|}
\hline S. No & Videos & PSNR dB \\
\hline 1 & Pepsi & 40 \\
\hline 2 & Lake [1] & 45 \\
\hline 3 & Foreman [7] & 46 \\
\hline
\end{tabular}




\section{International Journal of Science and Research (IJSR) \\ ISSN (Online): 2319-7064}

Index Copernicus Value (2013): 6.14 | Impact Factor (2015): 6.391

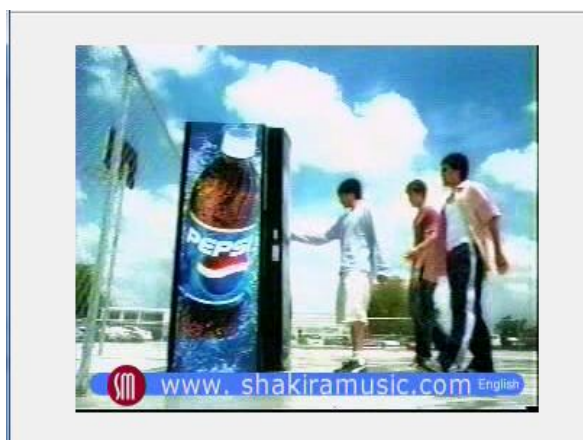

Figure 3 (a): Original Pepsi Video Frame

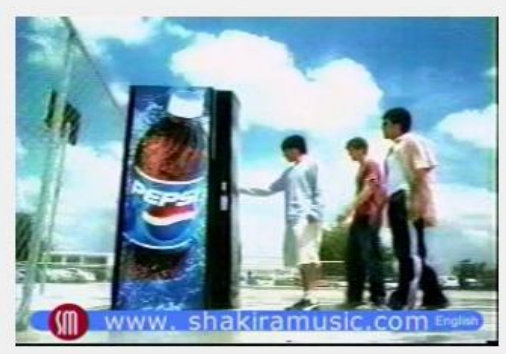

Figure 3 (b): Watermark Embedded Pepsi Video Frame [PSNR= 40]

\section{$A B C D$}

Figure 4 (a): Original Watermark

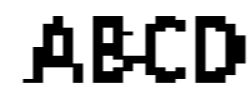

Figure 4 (b): Extracted Watermark

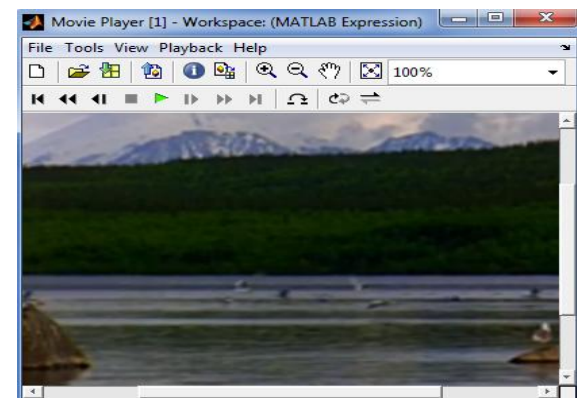

Figure 5: Lake video frame

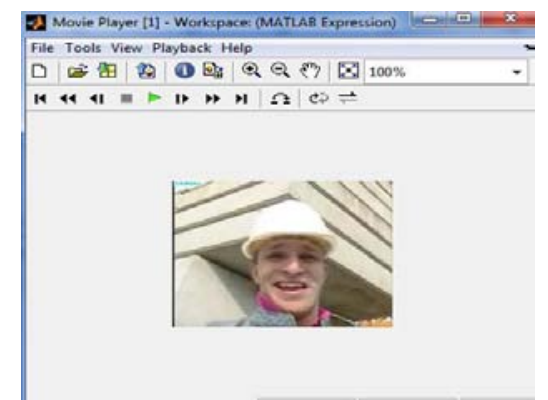

Figure 6: Foreman video frame

The original watermark and the extracted watermark shown in fig.4(a) and fig.4(b) in case of no attack. The $N C$ value of the watermark in this case is $\mathbf{0 . 9 7 3}$ this indicate the exact extraction. The PSNR for lake and foreman videos are also calculated by us which are shown in table 1 and the NC values for pepsi video after attack is shown in table 2 . From table 2 it is observed that after various attacks the watermark is extracted properly with slight difference with the original watermark.

Table 2: NC values for pepsi video

\begin{tabular}{|c|c|c|c|}
\hline Sr.no. & Attacks & Watermark after attack & NC value \\
\hline 1 & Speckle noise & $\mathrm{AB}$ & 0.97114 \\
\hline 2 & Gaussian noise & & 0.97823 \\
\hline 3 & $\begin{array}{l}\text { Histogram } \\
\text { equalization }\end{array}$ & ARED & 0.97936 \\
\hline 4 & Contrast adjustment & ht: & 0.97936 \\
\hline 5 & Cropping & hELD & 0.97936 \\
\hline 6 & $\begin{array}{c}\text { Salt and pepper } \\
\text { noise }\end{array}$ & hE & 0.96884 \\
\hline 7 & Poisson noise & & 0.97936 \\
\hline 8 & Frame dropping & AECD & 0.97936 \\
\hline 9 & Frame swapping & AECD & 0.97936 \\
\hline 10 & Frame averaging & hELD & 0.97936 \\
\hline 11 & JPEG compression & & 0.97936 \\
\hline 12 & Resizing & AECD & 0.97936 \\
\hline 13 & Rotation & HERD & 0.97936 \\
\hline
\end{tabular}

\section{Conclusion}

We have proposed the video watermarking scheme based on DWPT and PCA which is robust and imperceptible in nature. We have embedded the binary watermark in the low cA sub band which helped in increasing the robustness of the watermark against various common video processing attacks like noise attacks, Geometric attacks, Histogram equalization, contrast adjustment, frame dropping, frame averaging, frame swapping, etc. and the PCA algorithm helped in increasing the imperceptibility of the video. The results show that the proposed scheme is $12.5 \%$ and $18 \%$ better than the existing scheme [1] and [7] respectively, in terms of visual quality of video after embedding the watermark. So, the proposed video watermarking scheme based on DWPT and PCA satisfied the requirement of imperceptibility and robustness for a watermarking scheme.

\section{References}

[1] Sanjana Sinha, Prajnat Bardhan, Swarnali Pramanick, Ankul Jagatramka, Dipak K. Kole and Aruna Chakraborty, "Video Watermarking Scheme Based on Principal Component Analysis and Wavelet Transform", Department of Computer Science \& Engineering St Thomas' College of Engineering and Technology Kolkata, India. International Journal of Wisdom Based Computing, Vol. 1 (2), August 2011. 
[2] Alapan Arnab, Đigital Rights Management -A current review", Data Network Architecture Laboratory, Department of Computer Science, University of Cape Town, 2005.

[3] C.V. Serdean, M.A. Ambroze, M. Tomlinson and J.G. Wade, ĐWT Based Video Watermarking for Copyright Protection, Invariant to Geometrical Attacks", Proceedings of the $3^{\text {rd }}$ International Symposium on Communication Systems Networks and Digital Signal Processing - CSNDSP'02, Stafford, UK, 15-17 July 2002.

[4] Maher El'arbi, Chokri Amar and Henri Nicolas, "Video Watermarking Based on Neural Networks", IEEE International Conference on Multimedia and Expo, ICME'06, pp.1577-1580, 2006.

[5] Nisreen I. Yassin, Nancy M. Salem and Mohamed I. El Adawy, "Block Based Video Watermarking Scheme Using Wavelet Transform and Principle Component Analysis", IJCSI International Journal of Computer Science Issues, Vol. 9, Issue 1, No 3, January 2012.

[6] Salwa A. K. Mostafa, Naser El-Sheimy, A. S. Tolba, F. M. Abdelkader and Hisham M Elhindy, Wavelet Packets-Based Blind Watermarking for Medical Image Management", OBE Journal, Vol. 04, pp. 93-98, 2010.

[7] Salwa A.K Mostafa, A. S. Tolba , F. M. Abdelkader and Hisham M. Elhindy," Video Watermarking Scheme Based on Principal Component Analysis and Discrete wavelet transform", International Journal of Wisdom Based Computing, Vol. 1 (2), August 2010.

[8] Ankita A. Hood and Prof. N. J. Janwe, "A Hybrid \& Robust Wavelet Based Video Watermarking Scheme for Copyright Protection Using Principal Component Analysis", International Journal of Computer Trends and Technology (IJCTT) - volume 4 Issue 6-June 2013.

[9] Phadtare Saurabh, Dhebe Pooja, Bobade Sharayu and Jawalkar Nishigandha, "Video Watermarking Scheme based on DWT and PCA for Copyright Protection", IOSR Journal of Computer Engineering (IOSR-JCE), Volume 9, Issue 4, pp 2278-8727, Mar. - Apr. 2013.

[10] C. Patvardhan , A. K. Verma and C. Vasantha Lakshmi, "A Robust Wavelet Packet based Blind Digital Image Watermarking using HVS characteristics", International Journal of Computer Applications (0975 - 8887) Volume 36- No.9, December 2011.

\section{Author Profile}

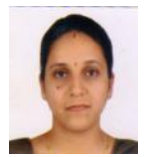

Ms. Shital Khaparde received the B.E. and M.E. degrees in Computer Science and Information Technology from Nagpur and Mumbai Universities in 2006 and 2014, respectively. 